\title{
SOFISTICAÇÃO DA PRODUÇÃO DOS ESTADOS BRASILEIROS: DETERMINANTES E IMPACTOS SOBRE O CRESCIMENTO ECONÔMICO.
}

\author{
Ariane Danielle Baraúna da Silva ${ }^{1}$ \\ Álvaro Barrantes Hidalgo ${ }^{2}$
}

\begin{abstract}
Resumo
O presente artigo estende o trabalho empírico de Hausmannet al. (2007) com o objetivo de examinar os efeitos da sofisticaçãosobre o crescimento econômico dos estados brasileiros,bem como os fatores que determinam a sofisticação. Segundo Hausmannet al. (2007) o incremento na sofisticação dos bens produzidos por um país tem impacto positivo sobre o crescimento econômico da região. Os resultados mostram que o crescimento econômico está diretamente ligado ao processo de desenvolvimento de bens mais sofisticados pelos estados brasileiros. As conclusões da pesquisa também sugerem que um dos principais determinantes da sofisticação é o capital humano,ou seja, os estados que possuem uma mão de obra mais qualificada tendem a exportar bens mais sofisticados. O investimento em pesquisa também tem um papel importante sobre a variável de interesse, esses resultados estão de acordo com o esperado, dado que essas duas variáveis representam a principal fonte de inovação nacional. Algumas variáveis apresentaram um efeito negativo sobre a sofisticação, como o grau de abertura comercial e a disponibilidade de recursos naturais.
\end{abstract}

Palavras-Chave:Crescimento Econômico, Sofisticação, Exportações.

\begin{abstract}
This article extends the empirical work of Hausmann et al. (2007) in order to examine the effects of sophistication on economic growth of the Brazilian states as well as the factors that determine the sophistication. According to Hausmann et al. (2007) the increase in the sophistication of goods produced by a country has a positive impact on economic growth in the region. The results show that economic growth is directly linked to the process of developing more sophisticated goods by states. The research findings also suggest that a major determinant of sophistication is the human capital, that is, states that have a more skilled labor tend to export more sophisticated goods. The investment in research also has an important role on the variable of interest, these results are consistent with the expected since these two variables represent the main
\end{abstract}

\footnotetext{
${ }^{1}$ Doutora em Economia (PIMES/UFPE).

${ }^{2}$ Professor Titular (Decon/UFPE).
} 
source of national innovation. Some variables had a negative effect on the sophistication, the degree of trade openness and the availability of natural resources.

Keywords:Economic Growth, Sophistication, Exports.

Classificação JEL: O11, O14, O30, O47, O54.

Área1: Indústria e Competitividade (1.3 - Crescimento, produtividade e competitividade).

\section{INTRODUÇÃO}

Estudos recentes têm mostrado que o crescimento econômico está diretamente ligado ao incremento na "sofisticação" dos bens produzidos por um país, em particular, de suas exportações, enfatizando que nem todos os produtos são iguais em termos de suas consequências para o crescimento econômico. Segundo esses autores ${ }^{3}$, a especialização em alguns produtos pode trazer maior crescimento. Essesbens podem produzir maiores spillovers de conhecimento, pois possuem um potencial maior para ligações para frente e para trás, ou oferecem um caminho mais fácil para outros produtos com taiscaracterísticas. Em última análise, alguns produtos são mais "sofisticados", no sentido de que eles estão associados com níveis de produtividade mais elevados, e os países que produzem tais bens tendem a apresentar melhor desempenho.

Setores sofisticados são particularmente propensos a agir como ummotor de crescimento para a economia, mas existem importantes pré-requisitos para o desenvolvimento de exportações sofisticadas, como uma força de trabalho qualificada e fluxos de informação,bem como uma política macroeconômica apropriada, esta desempenha um papel particularmente importante.

$\mathrm{O}$ presente artigo pretende analisar em que medida o incremento na sofisticação das exportações se traduz em crescimento econômico para os estados brasileiros, e os fatores que determinam a magnitude desse impacto, baseado nos trabalhos de Hausmannet.al. (2007) e Jarreau e Poncet (2009). Com esse objetivo, serão estimados dois modelo,o primeiro analisa o impacto da sofisticação das exportações sobre o crescimento econômico do Brasil, e o segundo procura detectar quais os principais determinantes da sofisticação dos produtos exportados pelo Brasil.

$\mathrm{O}$ resto do artigo está organizado da seguinte forma. A seção 2 descreve os principais trabalhos relacionados ao tema, aseção 3 apresenta a metodologia do estudo e descreve os dados utilizados e suas fontes. A seção 4 analisa empiricamente como a sofisticação das exportações afeta o crescimento econômico dos estados brasileiros e os fatores que determinam a sofisticação da produção, e por fim, a seção 5apresenta as principais conclusões do trabalho.

\section{REVISÃO DA LITERATURA}

Um número crescente de trabalhos tem se preocupado em analisar a importância da sofisticação da produção sobre o crescimento econômico,o objetivo da maioria desses

${ }^{3}$ Exemplo: Hausmann et. al. (2007), Lall, et al, (2005). 
estudos é testar a previsão de Hausmannet.al. (2007), segundo a qual o mix de produtos que um país produz pode ter implicações importantes para uma economia. Segundo essa literatura, nem todos os bens são similares em termos das suas consequências para o desempenho econômico, a especialização em alguns produtos traz um maior crescimento econômico.

Para testar a importância da sofisticação produtiva sobre o crescimento econômico,Hausmannet.al. (2007) constroem um índice de sofisticação baseado no nível de renda do país, em seguida estimam o impacto desse índice sobre o crescimento econômico de um conjunto de países. Para isso, utilizam uma regressão de crescimento acrescentando onível de sofisticação das exportações como preditor de crescimento subsequente. Os autores encontram um efeito positivo e significativo da sofisticação sobre o crescimento do $P I B$, indicando que de fato, os países que produzem bens mais sofisticados tendem a ter um melhor desempenho.

Jarreau e Poncet (2009) usam dados desagregados sobre o comércio da China durante o período de 1997 a 2007, para examinar a melhora recente das exportações chinesas e suas consequências sobre o desempenho econômico do país. Testam o impacto do aumento na sofisticação sobre o desempenho econômico das províncias Chinesas, usando como uma proxyo índice de sofisticação proposto porHausmannet.al. (2007).Os resultados indicam que a sofisticação das exportações influencia positivamente o desempenho econômico chinês. Também concluem que a estrutura de exportação de firmas estrangeiras não tem impacto direto sobre o crescimento econômico, mas tem um impacto indireto, por meio de sua influência positivana sofisticação de firmas nacionais, o que sugere a presença de spillovers.

$\mathrm{Na}$ parte final, investigam se os ganhos de especialização das exportações sofisticadas estão condicionadosa outras características das províncias, encontrando evidências de que esses ganhos são mais importantes nas províncias mais voltadas para o exterior, ou seja, com maior abertura comerciale aquelas que possuem um melhor acesso aos mercados externos. E por fim, encontram evidências de retornos crescentes para a sofisticação.

Dada sua aparente importância, alguns trabalhos tem se preocupam em estimar os fatores que determinam o incremento da sofisticação, Xuanet. al. (2007) analisam os determinantes da sofisticação das exportações de um grupo de países, abrangendo o período de 1992 a 2006. Os resultados mostram que a sofisticação das exportações é reforçada pela intensidade de capital, criação de conhecimento, investimento em educação, P\&D e investimento estrangeiro direto. Segundo os autores, o efeito da abundância de recursos naturais parece ser moderado pela qualidadedas instituições. $\mathrm{Na}$ ausência de instituições eficazesa abundância dificulta o avanço estrutural das exportações, no entanto, a existência de abundância pode exercer um impacto positivo onde existem boas instituições. Os efeitos dos determinantes variam entregrupos de paísescom diferentes níveis de renda.

Weldemicael (2012)examina o efeito causal do investimento estrangeiro direto (IED), do afastamento dos grandes mercados, e da qualidade institucional na sofisticação das exportações. Usando um painel para um grupo de países desenvolvidos, conclui que o investimento externo direto tem um efeito positivo, esse efeito é maior para os países combaixa qualidade institucional, segundo o autor a maior partedo investimento é feita em insumos e produtos primários, que não exigem um forte ambiente institucional. Da mesma forma, o afastamento dos grandes mercados tem um forte efeito negativo. Com relação à qualidade institucional, seuimpacto na sofisticação das exportações só é evidente no setor de manufaturados. 
A nível nacional, a literatura é bastante restrita, não se tem conhecimento de estudos mais aprofundados sobre o tema, como os citados acima, os trabalhos recentes como os de Kume (2008) e Costa (2014), se limitam a análises do índice de sofisticação sem nenhuma investigação explícita dos seus impactos sobre a economia brasileira, nem de seus determinantes. $\mathrm{O}$ presente ensaio pretende preencher essa lacuna, fazendo uma análise mais detalhada sobre o tema.

\section{METODOLOGIA E DADOS UTILIZADOS}

\subsection{Impacto da Sofisticação sobre o Crescimento Econômico dos Estados Brasileiros}

Nos últimos anos, algunstrabalhos têm desenvolvido índices para medir a sofisticação das exportações e examinar sua relação com o desenvolvimento tecnológico (Lall, et al., 2005; Rodrik, 2006; Hausmann, et al., 2007; Xu, 2010; Schott, 2008; Van Assche e Gangnes, 2008).Para tal fim, os autores constroem um índice de produtividade que chamam dePRODY. Esse índice é definido pela média ponderada da renda per capita dos países exportadores de determinado produto, representando o nível de renda associado a cada um deles. Cada país é indexado por $j$ e os produtos por $k$, sendoo total das exportações do país $j$ dado por:

$$
X_{j}=\sum_{k} x_{j k}
$$

Seja a renda per capita do país $j$ denotada por $Y_{j}$. Então o nível de produtividade associado ao produto $k$ é dado por:

$$
\operatorname{PRODY}_{k}=\sum_{j} \frac{\left(x_{j k} / X_{j}\right)}{\sum_{j}\left(x_{j k} / X_{j}\right)} Y_{j}
$$

Onde:

$P R O D Y_{k}$ é a produtividade do produto $k$;

$x_{j k}$ é o valor das exportações do produto $k$ pelo país $\mathrm{j} ; X_{j}$ é o valor das exportações totais do país $j$;

$Y_{j}$ é a renda per capita do país $j$

O numerador da fração, $x_{j k} / X_{j}$, é a participação do bem no total de produtos exportados pelo país $j$, o denominador, $\sum_{j}\left(x_{j k} / X_{j}\right)$, agrega as exportações de todos os países exportadores do bem $k$. Esse índice representa a média ponderada da renda per capita, onde a ponderação corresponde à vantagem comparativa revelada de cada país no bem $k$.

O ponderador da renda per capita é o indicador de vantagem comparativa revelada de Balassa (1965), normalizado para que a soma seja igual a $\mathrm{um}^{4}$.

${ }^{4} \mathrm{O}$ índice de vantagem comparativa revelada tradicional de Balassa (1965) mede, no denominador, a participação das exportações mundiais de um determinado produto no total das exportações do mundo. Assim, se a participação das exportações deste bem no total das exportações do país (numerador) for superior à parcela das exportações mundiais do mesmo produto no total exportado pelo mundo, o índice será maior que 1. Caso contrário, o índice será menor que 1 . Se o país j não exporta o bem $k$, o índice de 
Segundo Kume (2008), a ideia básica é que os países, ao exportarem um bem, "revelam" o seu grau de produtividade de forma similar ao conceito de vantagem comparativa revelada. Como os salários nos países ricos são mais elevados, as exportações serão viáveis somente se forem compensadas por meio de uma melhor tecnologia. Esta maior produtividade pode ser decorrente não apenas de uma tecnologia mais avançada, mas também de outros fatores, tais como a dotação de fatores, a infraestrutura, as técnicas de comercialização e a fragmentação da produção. Assim, pode-se atribuir uma medida de produtividade, denominada grau de sofisticação,aos produtos exportados para qualquer classificação de mercadorias. Segundo Lall, et al.(2005) a sofisticação se correlaciona muito bem com a tecnologia.

O Índice de sofisticação das exportações do país $j\left(E X P Y_{j t}\right)$, para cada anot é dada por:

$$
\operatorname{EXPY}_{j t}=\sum_{k} \frac{X_{j k t}}{X_{j t}} P R O D Y_{k}
$$

A expressão (3) representa a produtividade de cada produto $k$ ponderada por sua participação na pauta de exportação do paísj.

Nossa análise será realizada a nível estadual, sendo assim, obtemos um indicador de sofisticação anual para cada estado brasileiro.

O que se busca inicialmente é estimar o impacto da sofisticação das exportações sobre o crescimento econômico dos estados brasileiros. Segundo Hausmann, et. al. (2007), o crescimento econômico de um país depende, entre outros fatores, dos produtos que ele produz, países que se engajam no processo de desenvolvimento de produtos mais sofisticados devem ser capazes de capturar maiores ganhos do comércio, além daqueles associados àsvantagens comparativas tradicionais induzidaspela especialização. O nosso estudo irá proporcionar um teste desta teoria, estamos interessados em mostrar empiricamente que as regiões brasileiras que se especializam na produção de bens com níveis de produtividade mais elevados terão um desempenho melhor.

A metodologia do trabalho se baseou na estimação dos modelos descritos na Tabela 1, apresentada abaixo, os mesmos servirão como base para nosso estudo.

Tabela 1: Modelos Econométricos de Crescimento Econômicoa serem estimados.

\begin{tabular}{|c|c|}
\hline MODELO & ESPECIFICAÇÃO DO MODELO \\
\hline Mod I & $\log \left(y_{t}\right)=\alpha_{1}+\alpha_{2} E d+\alpha_{3} I+\alpha_{4} I I+\alpha_{5} A C+\alpha_{6} I P+\varepsilon$ \\
\hline Mod II & $\log \left(y_{t}\right)=\alpha_{1}+\alpha_{2} E d+\alpha_{3} I+\alpha_{4} I I+\alpha_{5} A C+\alpha_{6} I P+\alpha_{7} S E+\varepsilon$ \\
\hline Mod III & $\log \left(y_{t}\right)=\alpha_{1}+\alpha_{2} E d+\alpha_{3} I+\alpha_{4} T I+\alpha_{5} A C+\alpha_{6} I P+\alpha_{7} S E+\alpha_{7} S I+\varepsilon$ \\
\hline Mod IV & $\log \left(y_{t}\right)=\alpha_{1}+\alpha_{2} E d+\alpha_{3} I+\alpha_{4} T I+\alpha_{5} A C+\alpha_{6} I P+\alpha_{7} S E+\alpha_{7} S E^{2}+\varepsilon$ \\
\hline Mod $V$ & $\log \left(y_{t}\right)=\alpha_{1}+\alpha_{2} E d+\alpha_{3} I+\alpha_{4} T I+\alpha_{5} A C+\alpha_{6} I P+\alpha_{8} S \operatorname{Pr}+\alpha_{9} S M+\varepsilon$ \\
\hline
\end{tabular}

vantagem comparativa será nulo. $\mathrm{O}$ ajuste feito no índice permite que a soma dos índices de vantagem comparativa de todos os países seja igual a 1. 
No primeiro modelo são considerados os determinantes habituais do crescimento segundo os modelos tradicionais, que são: educação $(E d)$, taxa de investimento $(T I)$, infraestrutura $(I)$, grau de abertura comercial $(A C)$, investimento em pesquisa $(I P)$. $\mathrm{O}$ modelo $(I I)$ inclui, além dessas variáveis, o grau de sofisticação das exportações $(S E)^{5}$ dos estados brasileiros, podendo assim inferir sobre a importância do aprimoramento tecnológico das exportações sobre o desempenho econômico.

Verificado o impacto da sofisticação das exportações sobre o desempenho econômico, deve-se investigar se a relação entre as variáveis se deve a capacidade dos estados em melhorar a sofisticação dos produtos exportados ou simplesmente reflete o nível de sofisticação dos insumos anteriormente importados, ou seja, a estrutura de produção pode não corresponder completamente a estrutura das exportações. Com esse objetivo incluímos no modelo (IV) o nível de sofisticação dos produtos importados (SI), para mensurar a importância dessa variável sobre o desempenho econômico dos estados brasileiros.

Nopenúltimo modelo,analisamos se os benefícios da sofisticação sobre o crescimento estão aumentando com a extensão da sofisticação e do nível de renda, mostrandoum padrão de retornos crescentes, o que é captado pela inclusão do quadrado da sofisticação das exportações $(\mathrm{SE})^{2}$. O último modelo visa mensurar o impacto da sofisticação, separando a sofisticação agrícola $(S P r)$ da sofisticação de bens manufaturados $(S M)$, como exposto anteriormente, alguns autores sugerem que o impacto desta última variável é maior que o da primeira, o que se busca é verificar se tal argumento é válido ou não para o caso brasileiro.

\subsection{Determinantes da Sofisticação das Exportações brasileiras}

Nesta seção são apresentados os procedimentos metodológicos para estimação do efeito multiplicador da sofisticação sobre algumas variáveis econômicas. Seguir-se-á o modelo proposto por Hausmann, et al. (2007), onde postula-se uma economia com duas indústrias: umaindústria tradicional e uma indústria moderna. A indústria tradicional só produz bens homogêneos individuais, que são utilizados principalmente para o consumo interno, e a indústria moderna produz uma variedade de produtos. Em geral, a indústria moderna tem um nível de tecnologia relativamente alto. Postula-se que os fatores de produção básicos utilizados pela indústria moderna incluem recursos naturais, trabalho e capital físico. Assim, a função de produção nessa indústria é dada por:

$$
Y=A L^{\alpha} K^{\beta} N^{\gamma}(4)
$$

Onde L, K e $\mathrm{N}$ denotam os fatores de produção trabalho, capital, recursos naturais e A o nível tecnológicoda combinação de fatores de produção, respectivamente, onde a última variável exibe retornos constantes de escala, ou seja, $\alpha+\beta+\gamma=1$ e está distribuída uniformemente ao longo do intervalo $[0, \mathrm{~A}]$.

Seguindo Fagerberg (1998) e Sterlacchini (2008), admite-se uma função multiplicativa entre as fontes de conhecimento doméstico $(D)$, estrangeiro $(F)$, e a capacidade de colher os benefícios de ambos os tipos de conhecimento (I). A capacidade de explorar o conhecimento externo e interno é determinada pelas características institucionais, sociais e culturais de cada país. O acúmulo de

\footnotetext{
${ }^{5}$ Para tornar mais clara a apresentação das variáveis substituímos a nomenclatura usual, EXPY, por SE. Foi utilizada a mesma metodologia para a obtenção da sofisticação das importações (SI), dos Bens manufaturados (SM) e dos bens primários (SPr), alterando-se apenas os dados.
} 
conhecimentointerno normalmente é gerado pela pesquisa $(P \& D)$ e a educação; já o conhecimento estrangeiro é adquirido principalmente via investimento estrangeiro direto (IED) e importações. Omitindo sufixos da variável tempo, a função básica para o nível tecnológico Aé dada pela seguinte expressão:

$$
\hat{A}=B D^{\lambda_{D}} F^{\lambda_{F}} I^{\lambda_{I}}
$$

Onde $B$ incorpora o efeito de outros fatores que possivelmente afetam os parâmetros. $\lambda_{i}(i=D, F$ ou $I)$ representa as elasticidades de produção do conhecimento.Quanto maior $A$, maior a capacidade da economia em produzir bens de alta produtividade. As firmas podem imitar outros produtores sem incorrer em custos adicionais de "descoberta", mas em uma fração $\theta(0<\theta<1)$ de produtividade.

O modelo admite que cada investidor pode executar apenas um projeto. Assim, tendo desenvolvido seus próprios produtos, o investidor tem a escolha de continuar produzindo seus próprios bens ou imitando os produtos de mais alta produtividade. $\mathrm{O}$ investidor irá corrigir a sua escolha, comparando a produtividade $(A)$ de seus próprios bens ou dos produtos que imita. Se $A_{i}>\theta A^{\operatorname{Max}}$, o investidor escolhe ficar com o novo produto desenvolvido por ele mesmo, caso contrário vai imitar. Adicionalmente, as expectativas de $A^{M a x}$ dependem da fronteira de produtividade e do número $(m)$ de firmas que investem no setor moderno, que é dado por:

$$
E\left(A^{\operatorname{Max}}\right)=\frac{m A}{m+1}
$$

O parâmetro $\hat{A}$ é distribuído uniformemente sobre $[0, \hat{A}]$.

Segundo Hausmann, et al. (2007), a probabilidade e o nível técnico esperado do investidor que usa seus projetos sem imitar é:

$$
\begin{aligned}
& \operatorname{prob}\left(A_{i} \geq \theta A^{M a x}\right)=1-\frac{\theta m}{m+1} \\
& E\left(A_{i} / A_{i} \geq \theta A^{M a x}\right)=\frac{1}{2} \hat{A}\left[1+\frac{\theta m}{m+1}\right]
\end{aligned}
$$

Similarmente, a probabilidade e o nível técnico esperado para firmas que imitam os produtos $A^{\operatorname{Max}}$ são, respectivamente, os seguintes:

$$
\begin{array}{r}
\operatorname{prob}\left(A_{i}<\theta A^{\operatorname{Max}}\right)=\frac{\theta m}{m+1}(9) \\
E\left(A_{i} / A_{i}<\theta A^{M a x}\right)=\hat{A}\left[\frac{\theta m}{m+1}\right]
\end{array}
$$

Combinando essas equações, pode-se derivar o seguinte valor esperado para o parâmetro técnico $A$ no setor moderno:

$$
E(A)=\frac{1}{2} \hat{A}\left[1+\left(\frac{\theta m}{m+1}\right)^{2}\right]
$$

Introduzindo (2) em (1) e combinando a expressão resultante com (11), pode-se derivar o produto esperado como a seguir:

$$
E(Y)=\frac{1}{2} B\left[1+\left(\frac{\theta m}{m+1}\right)^{2}\right] D^{\lambda_{D}} F^{\lambda_{F}} I^{\lambda_{I}} K^{\beta} N^{\gamma}
$$


A expressão acima indica que a produção esperadada indústria moderna depende do capital, da disponibilidade de recursos naturais, do nívelde conhecimento interno e externo, da capacidade de assimilar ambos os tipos de conhecimento e do número de empresas que se dedicam ao desenvolvimento e descoberta de novos produtos.Postulase que a produção do setor moderno de exportação na economia, $E(Y)$, pode ser aproximada pelo índice de sofisticação $(E X P Y)$ utilizado anteriormente para quantificar a produtividade relativa das exportações.

Portanto, substituindo $E(Y)$ por $E X P Y$ na equação (12)e tirando o logaritmo, temos:

$$
\begin{aligned}
& \ln E X P Y_{i j t}=\beta_{0}+\beta_{1} \ln K_{i t}+\beta_{2} \ln N_{i t}+\beta_{3} \ln H_{i t}+\beta_{4} \ln P_{i t}+\beta_{5} \ln I E D_{i t} \\
& +\beta_{6} \ln M_{i t}+\beta_{7} \ln p o p_{i t}+v_{i}+u_{t}+\varepsilon_{i t}
\end{aligned}
$$

Onde:

$i$ representa o país, estado ou região exportadora;

$j$ denota o destino das exportações;

$t$ denota o período de tempo;

$v_{i}$ e $u_{t}$ o efeito fixo cross-section e o efeito fixo do período, respectivamente;

$\varepsilon_{i t}$ é o termo aleatório, que não está correlacionado com $v_{i}$ e $u_{t}$ ou com as variáveis independentes;

$K$ refere-se ao estoque de capital físico;

$N$ se refere aos recursos naturais, esta variável reflete a dotação dos recursos naturais de um país, patrimônio físico e força de trabalho relativa dos fatores. $H$ é capital humano, medido pela taxa de matrícula no ensino superior;

$P$ é a proporção de $P \& D$ sobre o PIB.

As duas últimas variáveis refletem o investimento em conhecimento interno de um país. IED e $M$ são a proporção anual de investimentos estrangeiros diretos e a proporção das importações de bens e serviços no PIB, respectivamente.Pop denota a população, usada comoproxy para o tamanho do estado.

\subsection{Dados Utilizados}

As estimações realizadas neste trabalho utilizam dados estaduais, a fim de considerar a heterogeneidade do país. O banco de dados foi construído com base em informações referentes às 26 unidades federativas do Brasil, acrescidas do Distrito Federal, totalizando 27 unidades de análise. O período contemplado pela análise compreende os anos de 2000 a 2010. A Tabela 2 apresenta uma síntese das variáveis consideradas no modelo, bemcomo suas proxiese fontes.

Tabela 2:Dados Utilizados para Construção das variáveis e no Modelo Econométrico.

\begin{tabular}{|l|l|c|}
\hline \multicolumn{1}{|c|}{ Variáveis Explicativas } & \multicolumn{1}{|c|}{ Proxy } & Fonte \\
\hline Investimento & Despesa de Capital Estadual. & IPEADATA \\
\hline Capital Humano & População Economicamente Ativa com 15 anos ou mais de Escolaridade. & IBGE \\
\hline Capial Físico & Consumo Industrial de Energia Elétrica & IBGE \\
\hline P\&D & Dispêndios dos governos estaduais em ciência e tecnologia (C\&T). & IPEADATA \\
\hline Abertura Comercial & Exportações mais Importações como proporção do PIB. & ALICEWEB \\
\hline Infraestrutura & Extensão Total, emquilômetros das rodovias, por Estado. & ANTT \\
\hline
\end{tabular}




\begin{tabular}{|l|l|c|}
\hline Exportações & Exportações Estaduais. & ALICEWEB \\
\hline Importações & Importações Estaduais. & ALICEWEB \\
\hline Export. Primárias & Exportações de Produtos Primários por Estado. & SECEX \\
\hline Export. de Manuf. & Exportações de Produtos Manufaturados por Estado. & SECEX \\
\hline
\end{tabular}

Fonte: Elaborada pelo Autor.

Os dados utilizados para realizar essas estimações terão várias fontes. Para o cálculo dos indicadores de sofisticação e orientação regional será feito uso da base de dados do Commodity Trade StatisticsDatabase (COMTRADE), no IBGE serão coletados os dados referentes à educação e a P\&D. As taxas de investimento $(T I)$ serão extraídas das séries históricas do IPEADATA e, por fim,utilizaremos dados do FINBRA (Finanças do Brasil) para construira proxy para o índice de infraestrutura, que será construída como uma média per capita das despesas estaduais dos setores estratégicos ${ }^{6}$.

As variáveis de cunho socioeconômico foram obtidas também através do portal IPEADATA e no site do Instituto Brasileiro de Geografia e Estatística (IBGE), no domínio IBGE Estados. Também foram utilizados microdados da Pesquisa Nacional de Amostra de Domicílios (PNAD). Utilizou-se a extensão total das rodovias estaduais como proxy para a infraestrutura, os dados foram extraídos do anuário estatístico de transportes terrestres, disponibilizados pela Agência Nacional de Transportes Terrestres (ANTT) e pelo portal estatístico do Instituto de Pesquisa Econômica Aplicada (IPEADATA).

Para o estoque de capital físico, utilizou-se como proxy a soma do consumo deenergia comercial e industrial obtidos junto a Ministério de Minas e Energia a partir doBalanço Energético Nacional (BEN), o qual é elaborado e divulgado anualmente pelaEmpresa de Pesquisa Energética (EPE). Os dados referentes à abertura comercial foram obtidos junto aos Serviços deComércio Exterior (SECEX) do Ministério de Desenvolvimento, Indústria e ComércioExterior (MDIC). Como indicador de abertura comercial foi utilizada a razão entre a soma daexportação e importação por fator agregado pelo PIB ((Importação + Exportação)/PIB).

\section{RESULTADOS OBTIDOS}

Como apresentando na seção anterior, esse ensaio tem dois objetivos principais: (I) estimar a importância da sofisticação das exportações brasileiras no crescimento econômico dos estados brasileiros, e (II) Mensurar os determinantes da sofisticação, buscando verificar quais variáveis tem maior impacto sobre a mesma. Com esse objetivo serão estimados dois modelos utilizando a técnica de dados em painel, uma vez que a base de dados reúne tanto observações de cross-section quanto séries de tempo.

A utilização desse método nos permite controlar a heterogeneidade não observadapresente nas relações bilaterais de comércio. SegundoWooldridge (2002), caso a heterogeneidade não observada não se correlacione com os regressores da equação, o método de Mínimos Quadrados (MQO/ Pooled)fornece estimativas não viesadas e consistentes dos parâmetros. No entanto, segundo o autor, estimativas mais eficientes podem ser obtidas por meio do método de Efeitos Aleatórios (RandomEffects$R E)$. Caso a heterogeneidade não observada esteja correlacionada com os regressores da equação, os parâmetros obtidos a partir do método MQO (Pooled) e os obtidos por Efeitos Aleatórios (RandomEffects-RE) serão viesados e inconsistentes. Neste caso, os

\footnotetext{
${ }^{6}$ São consideradas como estratégicas as áreas de atuação prioritárias da economia, por exemplo: saúde, educação, energia e transporte.
} 
parâmetros podem ser estimados de maneira não viesada e consistente por meio de Efeitos Fixos (FixedEffect-FE).

Para decidir entre o modelo de efeitos fixos e efeitos aleatórios foi utilizado o teste de Hausman, cuja hipótese alternativa indica que o modelo de efeitos aleatórios não é consistente, pois os coeficientes do modelo e os efeitos aleatórios não são ortogonais. $\mathrm{O}$ teste realizado mostrou que é possível rejeitar a hipótese nula, levando a concluir que o modelo de efeitos aleatórios não seria adequado. Ademais, o teste de Breusch-Pagan conclui pela presença de efeitos individuais significativos.

Os resultados mostram que não é possível ignorar a presença de efeitos significativos, estes só podem ser tratados adequadamente através do estimador de Efeitos Fixos. Sendo assim, os coeficientes reportados pelos estimadores de efeitos aleatórios e poolingsão considerados apenas a título de comparação. Os resultados dos testes são apresentados na Tabela 3, a seguir.

Tabela 3: Testes Estatísticos para escolha do melhor modelo e do método de estimação.

\begin{tabular}{l|cccccc}
\hline & Modelo I & Modelo II & Modelo III & Modelo IV & Modelo V & Modelo VI \\
\hline Teste Breusch-Pagan & 1626,13 & 1710,13 & 1745,13 & 1511,31 & 1419,63 & 1619,11 \\
Prob $>$ chi2 & $(0,00)$ & $(0,00)$ & $(0,00)$ & $(0,00)$ & $(0,00)$ & $(0,00)$ \\
\hline Teste de Hausman & 145,33 & 242,17 & 157,17 & 168,13 & 261,23 & 365,41 \\
Prob $>$ chi2 & $(0,00)$ & $(0,00)$ & $(0,00)$ & $(0,00)$ & $(0,00)$ & $(0,00)$ \\
\hline
\end{tabular}

Antes de estimar o modelo foi investigada a existência de multicolinearidade entre as variáveis, na presença de multicolinearidade severa os coeficientes podem não refletir o efeito particular da variável explicativa sobre aregressora, pois o coeficiente estará contaminadopelo efeito de outras variáveis e apenas um efeito parcial lhe poderá ser atribuído. Com o intuito de verificar se efetivamente existe a presença de multicolinearidade no modelo, foicalculado o fator de inflação da variância (FIV), a literatura considera que existemulticolinearidade severa quando o FIV>10. O FIV foi inferior a 5 para todas as variáveis, assim, o modelo foi gerado sem a exclusão de qualquer das variáveis aqui analisadas.

Tentando superar os prováveis problemas de heterocedasticidade e de autocorrelação todos os modelos foram gerados ajustando a matriz de variâncias para a forma robusta. O primeiro modelo apresenta a regressão de crescimento antes da adição do índice de sofisticação das exportações $(S E)$ dos estados brasileiros, esse modelo servirá para mensurar os impactos da introdução dessa nova variável que é apresentado no segundo modelo. No terceiro modelo, adicionamos o quadrado da sofisticação com o intuito de verificar se há algum padrão de retornos crescentes dessa variável sobre o PIB dos estados brasileiros. Os resultados do primeiro modelo são apresentados na Tabela 4 a seguir.

Tabela 4: Regressão de Crescimento Estadual (2000-2010).

\begin{tabular}{|c|c|c|c|c|c|c|c|c|c|}
\hline \multicolumn{10}{|c|}{ Variável Dependente:In do PIBEstadual } \\
\hline \multirow{2}{*}{$\begin{array}{c}\text { Variáveis } \\
\text { Independentes }\end{array}$} & \multicolumn{3}{|c|}{ Modelo I } & \multicolumn{3}{|c|}{ Modelo II } & \multicolumn{3}{|c|}{ Modelo III } \\
\hline & Pooled & Efeito Aleatório & Efeito Fixo & Pooled & Efeito Aleatório & Efeito Fixo & Pooled & Efeito Aleatório & Efeito Fixo \\
\hline \multirow{2}{*}{ lnInvest. } & $0,007 * *$ & $0,039 *$ & $0,047 *$ & $0,001 * *$ & $0,040^{*}$ & $0,049 *$ & $0,016^{*}$ & $0,031^{*}$ & $0,046^{*}$ \\
\hline & $(2,93)$ & $(6,55)$ & $(5,54)$ & $(2,09)$ & $(5,01)$ & $(5,65)$ & $(4,68)$ & $(6,46)$ & $(5,31)$ \\
\hline \multirow[t]{2}{*}{$\ln \mathrm{Edu}$} & $0,052 *$ & $0,312 *$ & $0,285^{*}$ & $0,065^{*}$ & $0,355^{*}$ & $0,288^{*}$ & $0,085^{*}$ & $0,321 *$ & $0,298^{*}$ \\
\hline & $(15,18)$ & $(13,18)$ & $(17,83)$ & $(15,43)$ & $(11,43)$ & $(17,84)$ & $(15,33)$ & $(15,33)$ & $(17,55)$ \\
\hline
\end{tabular}




\begin{tabular}{|c|c|c|c|c|c|c|c|c|c|}
\hline \multirow{2}{*}{$\ln \mathrm{P} \& \mathrm{D}$} & $-0,005^{\mathrm{NS}}$ & $-0,003^{\mathrm{NS}}$ & $0,007 * *$ & $-0,006^{\mathrm{NS}}$ & $-0,006^{\mathrm{NS}}$ & $0,007 * *$ & $-0,008^{\mathrm{NS}}$ & $-0,008^{\mathrm{NS}}$ & $0,006 * *$ \\
\hline & $(-0,64)$ & $(-0,23)$ & $(1,39)$ & $(-0,78)$ & $(-0,45)$ & $(1,37)$ & $(-1,06)$ & $(-1,02)$ & $(1,34)$ \\
\hline \multirow{2}{*}{ ln Abert.Com. } & $1,042 *$ & $1,048 *$ & $0,933 *$ & $1,124 *$ & $0,982 *$ & $0,959 *$ & $1,094 *$ & $1,033 *$ & $0,935^{*}$ \\
\hline & $(4,04)$ & $(4,68)$ & $(5,29)$ & $(4,27)$ & $(5,10)$ & $(5,41)$ & $(4,12)$ & $(5,12)$ & $(5,28)$ \\
\hline \multirow{2}{*}{ lninfraest. } & $0,002 * * *$ & $0,001 * * *$ & $-0,005^{\mathrm{NS}}$ & $0,027^{\mathrm{NS}}$ & $0,022^{\mathrm{NS}}$ & $-0,008^{\mathrm{NS}}$ & $0,034 * * *$ & $0,014 * * *$ & $-0,004^{\mathrm{NS}}$ \\
\hline & $(1,43)$ & $(1,65)$ & $(-0,32)$ & $(1,22)$ & $(1,32)$ & $(-0,47)$ & $(1,50)$ & $(1,48)$ & $(-0,23)$ \\
\hline \multirow{2}{*}{$\ln \mathrm{SE}$} & - & - & - & $0,055^{*}$ & $0,141 *$ & $0,162 * *$ & $0,037 * *$ & $0,128 * *$ & $0,139 *$ \\
\hline & - & - & - & $(4,47)$ & $(4,56)$ & $(2,30)$ & $(2,13)$ & $(2,21)$ & $(4,03)$ \\
\hline \multirow[t]{2}{*}{$\ln \mathrm{SE}^{2}$} & - & - & - & - & - & - & $-0,021 * * *$ & $-0,041 * * *$ & $-0,012 * * *$ \\
\hline & - & - & - & - & - & - & $(-1,41)$ & $(-1,50)$ & $(-1,81)$ \\
\hline $\mathrm{R}^{2}$ & 0,94 & 0,90 & 0,92 & 0,96 & 0,92 & 0,97 & 0,96 & 0,92 & 0,98 \\
\hline Obs. & 297 & 297 & 297 & 297 & 297 & 297 & 297 & 297 & 297 \\
\hline
\end{tabular}

Os resultados do primeiro modelo sugerem um impacto positivo do investimento, da educação e do grau de abertura comercial, todos foram estatisticamente significativos a $1 \%$. O impacto do investimento (Invest.) esteve em torno de $0,04 \%$, a educação $(E d u)$ apresentou um efeito mais forte, girando em torno de $0,30 \%$, o grau de abertura $(A C)$ apresentou maior impacto sobre o crescimento do PIB, este ficou em torno de $1,04 \%$ nos modelos pooled e efeitos aleatórios, e próximos a $1 \%$ no modelo utilizando efeitos fixos.

$\mathrm{O}$ coeficiente doinvestimento em pesquisa $(P \& D)$ foi positivo e significante no modelo de efeitos fixos, embora mostre um impacto de apenas $0,006 \%$. O estoque de infraestrutura apresentou um impacto negativo e não significativo nos modelos de efeitos fixos. Analisando os dados usados como proxy para infraestrutura ${ }^{7}$ percebe-se que não houve uma evolução satisfatória dessa variável, em alguns casos a mesma apresentou um comportamento decrescente, o que de fato prejudica a evolução do crescimento econômico, representando um entrave para o mesmo.

Partindo para o objeto de nosso estudo, introduzimos a sofisticação das exportações $(S E)$ como variável explicativa no modelo $I I$, os resultados mostram que há uma relação positiva e entre sofisticação e PIB estadual nos em todos os modelos estimados, sendo estatisticamente significante em todos eles. De acordo com os resultados, um aumento de $1 \%$ na sofisticação das exportações estaduais eleva em aproximadamente $0,15 \%$ o PIB dos estados brasileiros. Esses resultados sugerem que a especialização em produtos mais sofisticados traz retornos positivos em termos de crescimento para as regiões brasileiras, sugerindo que os estados com estrutura de produção mais sofisticada podem atingir taxas de crescimento mais elevadas.

Esse impacto foi maior que o encontrado para outros países como em Jarreau e Poncet (2011), os autores encontram um valor de 0,07\% para o mesmo coeficiente, enquanto em Hausmam, et al. (2007) esse valor gira em torno de $0,05 \%$ no modelo com dados cross-section, com dados em painel, o coeficiente varia entre $0,02 \%$ e $0,09 \%$, os dois trabalhos são aplicados à economia Chinesa.

O terceiro modelo inclui o quadrado da sofisticação $\left(S E^{2}\right)$ entre as variáveis explicativas, nosso objetivo é verificar se os benefícios da sofisticação sobre o PIB regional estão aumentando com a extensão da sofisticação, mostrandoum padrão de retornos crescentes. Analisando os resultados, não é possível comprovar tal

\footnotetext{
${ }^{7}$ Quilômetros de rodovias pavimentadas.
} 
comportamento, uma vez que os coeficientes da variável quadrática apresentaram valores negativos na maioria dos modelos.

O próximo modelo pretende investigar se a relação entre as variáveis se deve a capacidade interna dos estados em melhorar a sofisticação dos produtos exportados ou simplesmente reflete o nível de sofisticação dos insumos anteriormente importados, ou seja, a estrutura de produção pode não corresponder completamente à estrutura das exportações, a questão é até que ponto a sofisticação das exportações é simplesmente o reflexodonível de sofisticação dos insumos anteriormente importados. Com esse objetivo incluímos no modelo o nível de sofisticação dos produtos importados (SI). Os resultados são apresentados na Tabela 5, a seguir.

Tabela 5: Regressão de Crescimento Estadual (2000-2010).

\begin{tabular}{|c|c|c|c|c|c|c|}
\hline \multicolumn{7}{|c|}{ Variável Dependente:In do PIBEstadual } \\
\hline \multirow{2}{*}{$\begin{array}{c}\text { Variáveis } \\
\text { Independentes }\end{array}$} & \multicolumn{3}{|c|}{ Modelo IV } & \multicolumn{3}{|c|}{ Modelo V } \\
\hline & Pooled & Efeito Aleatório & Efeito Fixo & Pooled & Efeito Aleatório & Efeito Fixo \\
\hline \multirow[t]{2}{*}{ lnInvest. } & $0,007 * *$ & $0,035^{*}$ & $0,048 *$ & $0,009 * *$ & $0,033^{*}$ & $0,047 *$ \\
\hline & $(2,12)$ & $(4,22)$ & $(5,62)$ & $(2,06)$ & $(5,26)$ & $(5,59)$ \\
\hline \multirow[t]{2}{*}{$\ln \mathrm{Edu}$} & $0,066^{*}$ & $0,312^{*}$ & $0,286^{*}$ & $0,031 *$ & $0,265^{*}$ & $0,284 *$ \\
\hline & $(15,09)$ & $(13,09)$ & $(17,24)$ & $(15,70)$ & $(14,12)$ & $(17,73)$ \\
\hline \multirow[t]{2}{*}{$\ln \mathrm{P} \& \mathrm{D}$} & $-0,006^{\mathrm{NS}}$ & $-0,004^{\mathrm{NS}}$ & $0,007 * *$ & $-0,005^{\mathrm{NS}}$ & $-0,006^{\mathrm{NS}}$ & $0,008^{* *}$ \\
\hline & $(-0,79)$ & $(-0,54)$ & $(1,39)$ & $(-0,62)$ & $(-1,84)$ & $(1,62)$ \\
\hline \multirow[t]{2}{*}{$\ln \mathrm{AC}$} & $1,112 *$ & $0,812^{*}$ & $0,969^{*}$ & $1,085^{*}$ & $1,022^{*}$ & $0,917 *$ \\
\hline & $(4,20)$ & $(5,20)$ & $(5,42)$ & $(4,19)$ & $(4,99)$ & $(5,23)$ \\
\hline \multirow[t]{2}{*}{ lnInfraest. } & $0,003 * *$ & $0,002 * *$ & $-0,007^{\mathrm{NS}}$ & $0,052 * *$ & $0,038 * *$ & $-0,013^{\mathrm{NS}}$ \\
\hline & $(1,18)$ & $(1,95)$ & $(-0,54)$ & $(2,17)$ & $(1,76)$ & $(-0,76)$ \\
\hline \multirow[t]{2}{*}{$\ln \mathrm{SE}$} & $0,010 * *$ & $0,085^{*}$ & $0,050^{*}$ & - & - & - \\
\hline & $(2,32)$ & $(4,17)$ & $(6,20)$ & - & - & - \\
\hline \multirow[t]{2}{*}{$\ln \mathrm{SI}$} & $0,038^{*}$ & $0,029 *$ & $0,034 *$ & - & - & - \\
\hline & $(6,34)$ & $(6,89)$ & $(7,66)$ & - & - & - \\
\hline \multirow[t]{2}{*}{$\ln \mathrm{SPr}$} & - & - & - & $0,044^{*}$ & $0,021^{*}$ & $0,016^{*}$ \\
\hline & - & - & - & $(6,89)$ & $(6,21)$ & $(5,21)$ \\
\hline \multirow[t]{2}{*}{$\ln \mathrm{SM}$} & - & - & - & $0,018 * * *$ & $0,011 *$ & $0,007 * *$ \\
\hline & - & - & - & $(0,96)$ & $(3,61)$ & $(2,61)$ \\
\hline $\mathrm{R}^{2}$ & 0,95 & 0,93 & 0,95 & 0,95 & 0,96 & 0,96 \\
\hline Obs. & 297 & 297 & 297 & 297 & 297 & 297 \\
\hline
\end{tabular}

Fonte: Resultados obtidos pela autora com dados da pesquisa através do Programa Stata 11.0. *Estatisticamente significante a $1 \%, * *$ estatisticamente significante a $5 \%, * * *$ estatisticamente significante a $10 \%$, NS não significante estatisticamente.

Os resultados mostram que a sofisticação das importações é uma variável significantena determinação da renda dos estados brasileiros em todos os modelos estimados, de acordo com os resultados um aumento de $1 \%$ na sofisticação das importações eleva em aproximadamente $0,03 \%$ o PIB dos estados brasileiros. Com a introdução dessa nova variável os coeficientes do (SE) são reduzidos, sugerindo que parte do efeito deste último sobre o PIB se devia à sofisticação das importações. Esses resultados sugerem que o impacto positivo da sofisticação se deve em parte a entrada de bens intermediários mais sofisticados.

Os resultados de Jareau e Poncet (2009) para a economia chinesa foram estatisticamente insignificantes, indicando segundo o autor, que a sofisticação da cesta 
de importações não fornece nenhuma explicação adicional para o crescimento econômico das províncias chinesas.

O último modelo visa mensurar o impacto da sofisticação das exportações, separando em bens primários ( $S P r)$ e manufaturados $(S M)$, como exposto anteriormente, uma parte da literatura sugere que o impacto desta última variável é maior, o que se busca é verificar se tal argumento é válido para o caso brasileiro. Analisando os resultados, percebe-se que os mesmos vão contra essa proposição, a evolução da sofisticação de setores primários apresenta impacto maior do que os bens manufaturados em todas as estimações, sendo todos estatisticamente significantes.

Dada a importância da sofisticação no desempenho econômico regional brasileiro, verificada nas estimações acima, nosso próximo passo é mensurar quais são os principais determinantes dessa variável, assim será possível identificar a melhor forma de estimular o crescimento da mesma, dando subsídios á formulação de políticas econômicas para o comércio exterior. Para isso, usamos a sofisticação das exportações como variável dependente e introduzimos alguns de seus possíveis determinantes, conforme sugerido pela literatura. Os resultados são apresentados na Tabela 6, a seguir.

Tabela 6: Determinantes da Sofisticação das Exportações (2000-2010).

\begin{tabular}{|c|c|c|c|}
\hline \multicolumn{4}{|c|}{ Variável Dependente:Inda Sofisticação } \\
\hline \multirow{2}{*}{$\begin{array}{c}\text { Variáveis } \\
\text { Independentes } \\
\end{array}$} & \multicolumn{3}{|c|}{ Modelo VI } \\
\hline & Pooled & Efeito Aleatório & Efeito Fixo \\
\hline \multirow[t]{2}{*}{ lnCapital Físico } & $0,055^{* *}$ & $0,047 *$ & $0,081^{*}$ \\
\hline & $(2,60)$ & $(3,88)$ & $(3,94)$ \\
\hline \multirow[t]{2}{*}{$\ln$ Recursos Naturais } & $-0,096 * *$ & $-0,079 * *$ & $-0,113 * *$ \\
\hline & $(-2,71)$ & $(-2,12)$ & $(-2,80)$ \\
\hline \multirow[t]{2}{*}{ InCapital Humano } & $0,011 * *$ & $0,122^{*}$ & $0,145^{*}$ \\
\hline & $(2,87)$ & $(3,91)$ & $(4,31)$ \\
\hline \multirow[t]{2}{*}{$\ln \mathrm{P} \& \mathrm{D}$} & $0,030 * *$ & $0,051 * *$ & $0,126 * *$ \\
\hline & $(2,15)$ & $(2,22)$ & $(2,80)$ \\
\hline \multirow[t]{2}{*}{ lnInvest. } & $-0,018^{\mathrm{NS}}$ & $-0,009^{\mathrm{NS}}$ & $0,013 * *$ \\
\hline & $(-1,83)$ & $(-1,22)$ & $(2,32)$ \\
\hline \multirow[t]{2}{*}{ InPopulação } & $0,178 * *$ & $0,124 * *$ & $0,710^{* *}$ \\
\hline & $(2,68)$ & $(2,45)$ & $(2,27)$ \\
\hline \multirow[t]{2}{*}{ In Abert. Com. } & $-0,369 * *$ & $-0,341 * * *$ & $-0,691 * *$ \\
\hline & $(-2,34)$ & $(-1,23)$ & $(-2,31)$ \\
\hline$R^{2}$ & 0,44 & 0,48 & 0,55 \\
\hline Obs. & 297 & 297 & 297 \\
\hline
\end{tabular}

Os resultados mostram uma relação positiva e estatisticamente significante entre sofisticação e capital físico, seu impacto é mais forte no modelo de efeitos fixos, onde um aumento de $1 \%$ no capital físico eleva em aproximadamente $0,08 \%$ a sofisticação. Como mencionado anteriormente, foi usado o consumo de energia elétrica como proxy para o capital físico, segundo alguns autores essa variável pode subestimar os resultados, dado que novas tecnologias podem permitir um maior nível de produção, 
com menor consumo de energia, mas diante da impossibilidade de obter dados estaduais para o capital físico, essa variável tem sido utilizada como proxy na maioria dos modelos estaduais de crescimento.

O coeficiente da variável que mensura a disponibilidade de recursos naturais foi negativo e estatisticamente significante nos modelos estimados, suportando a hipótese da "maldição dos recursos naturais", segundo a qual a abundância desse fator dificulta a acumulação de competências e o aperfeiçoamento da indústria. Alguns autores como Bresser Pereira (2008), têm mostrado que economias abundantes em recursos naturaistendem a crescer mais lentamente do que as economias sem recursos naturais substanciais. Esses resultados corroboram com Hausmamet at.(2007), o autor analisou a relação entre sofisticação das exportações e disponibilidade de recursos naturais para uma amostra de países, verificando que os países com maior disponibilidade de recursos apresentam menor sofisticação das exportações.

Associando esse resultado com os obtidos no modelo cinco, que introduz o grau de sofisticação do setor primário (SPr), pode-se inferir que embora a disponibilidade de recursos naturais afete negativamente a sofisticação das exportações brasileiras, 0 incremento na sofisticação de bens mais intensivos em recursos naturais pode gerar um impacto maior no crescimento quando comparado ao setor de bens manufaturados. $\mathrm{O}$ primeiro resultado pode estar relacionado à maior dificuldade de se aprimorar esses bens. No entanto, para se afirmar com maior segurança seria necessário uma análise com maior nível de desagregação setorial.

O capital humano e o investimento em $\mathrm{P} \& \mathrm{D}$ são as fontes principais de inovação nacional, e como era esperado apresentam um impacto positivo sobre a sofisticação das exportações brasileiras, os coeficientes foram estatisticamente significantes em todos os modelos estimados, de acordo com os resultados para o modelo de efeitos fixos, um aumento de $1 \%$ no capital humano gera um crescimento de aproximadamente $0,15 \%$ na sofisticação, para o investimento em $\mathrm{P} \& \mathrm{D}$ o incremento seria de aproximadamente $0,13 \%$.

O capital humano é uma das variáveis de maior impacto no modelo, este resultado corrobora com a importância do investimento em capital humano para o crescimento do país e para atenuar as suas disparidades regionais proposta por uma vasta literatura. Diversos autores como Nelson e Phelps (1966), Lucas (1988), Romer (1990) e Mankiw, Romer e Weil (1992) e Ferreira, Issler e Pessoa (2004),têm enfatizado a importância dessa variável, e mostram que o impacto da mesma supera à do capital físico e de outras variáveis importantes para a explicação do crescimento econômico.

O investimento, por sua vez, apresentou coeficiente negativo para a estimação poolede de efeitos aleatórios, e positivo para o modelo de efeitos fixos, sendo estatisticamente significante apenas no último, de acordo com o mesmo, um aumento de $1 \%$ no investimento provoca um crescimento de aproximadamente $0,013 \%$ na sofisticação das exportações estaduais. O coeficiente da população, usado como proxy para o tamanho do estado e força econômica, exerce um impacto positivo na sofisticação das exportações.

Por último, foi incluído no modelo o índice de abertura comercial, seu coeficiente apresentou um valor negativo e relativamente alto, mostrando que o aumento de $1 \%$ na abertura comercial provoca uma redução de aproximadamente $0,69 \%$ de acordo com o modelo de efeitos fixos, esse resultado é corroborado por alguns estudos $^{8}$, como por exemplo, Bastos, et. al. (2011) mostra que um aumento de $1 \%$ na

\footnotetext{
${ }^{8}$ Destacam-se os trabalhos de Kim e Lin (2009), Kiyota (2009), Dowrick e Golley (2004), Freund e Bolaky (2008), Herzer (2011), Bertella e Lima (2004), Grossman e Helpman (1990, 1991).
} 
abertura comercial gera um decréscimo de $0,07 \%$ na renda per capita dos estados brasileiros, esses estudos argumentam que as disparidades regionais, a incapacidade de absorção de novas tecnologias e as distorções que o comércio provoca nos termos de troca fazem com que a abertura comercial passe a afetar negativamente as economias.

\section{CONCLUSÕES}

Nos últimos anos, um número crescente de trabalhos tem se dedicado à construção de índices para medir a sofisticação das exportações. No entanto, os seus determinantes não foram totalmente explorados até agora, a literatura internacional ainda conta com um reduzido, mas crescente número de trabalhos explorando esse tema, em termos nacionais não se tem conhecimento até o momento de tal análise, apenas alguns poucos trabalhos fazendo uma análise descritiva dos índices de sofisticação.

O presente trabalho tenta preencher esta lacuna através da estimação do impacto dos incrementos na sofisticação das exportações estaduais para o desempenho da economia brasileira, à luz das teorias de comércio internacional. Através da estimação com dados em painel para o período de 1992 a 2006, e fazendo uso das variações regionais dentro de um único país, investigamos se a sofisticação das exportações exerce algum impacto sobre o crescimento econômico dos estados brasileiros. Os resultados confirmam a previsão de Hausmann, et. al.(2007), de que as regiões que se engajam no processo de desenvolvimento de produtos mais sofisticados captam maiores ganhos da globalização e tem um crescimento econômico mais rápido.

Os resultados mostraram queo crescimento econômico está diretamente ligado ao processo de desenvolvimento de bens com maior grau de sofisticação, podemos afirmar que a sofisticação das exportações gera impactos positivos sobre a renda dos estados brasileiros, ou seja, as regiões que se envolvem no processo de desenvolvimento de produtos mais sofisticados captam maiores ganhos da globalização gerando impactos positivos sobre sua renda, confirmando a previsão de Hausmann, et. al. (2007). Também foi possível concluir que a sofisticação não tem apresentado retornos crescentes para a economia brasileira e que a sofisticação dos bens pertencentes ao setor primário tem gerado maiores retornos quando comparado ao setor de bens manufaturados.

Dada a importância da sofisticação para o desempenho econômico regional, sugerido pelos resultados desta pesquisa, o segundo passo foi mensurar seus determinantes. As conclusões da pesquisa sugerem que o capital humano é um importante impulsionador da sofisticação, os estados que possuem uma mão de obra mais qualificada tendem a exportar bens mais sofisticados. $\mathrm{O}$ investimento em pesquisa também tem um papel importante sobre a variável de interesse. Esses resultados estão de acordo com o esperado, dado que essas duas variáveis representam a principal fonte de inovação nacional.

Por outro lado, algumas variáveis apresentaram um efeito negativo sobre a sofisticação, como o grau de abertura comercial e a disponibilidade de recursos naturais nos estados brasileiros, esses resultados foram consistentes com a literatura. Segundo alguns autores, como Mehlumet. al. (2006), o impacto negativo dessas variáveis, em especial a abundância de recursos naturais, é moderado quando há uma qualidade institucional, a importância dessa variável para a sofisticação é confirmada no trabalho de Weldemicael(2012), o que mostra o importante papel do governo sobre o aperfeiçoamento da sofisticação, podendo intensificar o impacto positivo de algumas variáveis, bem como minimizar os impactos negativos de outras. 
Os resultados apresentados neste trabalho mostram que a sofisticação tem um impacto significativo sobre a renda dos estados brasileiros, desse modo, o aperfeiçoamento desse mecanismo propagador do crescimento brasileiro deve ser alvo de políticas governamentais que estimulem seu desenvolvimento, e como apresentado acima, isso pode ser feito de forma mais efetiva através do investimento em capital humano e $P \& D$, uma vez que essas variáveis mostraram ter uma forte influência sobre a sofisticação das exportações brasileiras.

\section{REFERÊNCIAS BIBLIOGRÁFICAS}

Bastos, et. al. Abertura Comercial e Nível de Renda dos Estados Brasileiros: Uma Análise com Modelos Dinâmicos de Dados em Painel. XVI Encontro Regional de Economia, 2011.

Bresser Pereira, C. Doença holandesa e sua neutralização: uma abordagem ricardiana", in Doença holandesa e a indústria, Editora da Fundação Getúlio Vargas, 2008.

Costa, K. Fragmentação internacional da produção e sofisticação das exportações: uma análise a partir dos fluxos de comércio do Mercosul entre 1994 e 2012. XXXXII Encontro Nacional de Economia, 2014.

Weldemicael. Determinants of Export Sophistication. University of Melbourne, 2012.

Jeffrey M. Wooldridge Econometric Analysis of Cross Section and Panel Data. The MIT Press. Cambridge, Massachusetts. London, England, 2002.

Fagerberg, J. 'Why Growth Rates Differ', In: Dosi, G., Freeman, C., Nelson, R., Silverberg, G., Soete, L. (Eds.), Technical Change and Economic Theory. Pinter Publishers, London, 1998.

Ferreira, P. C.; Rossi, J. L. New Evidence from Brazil on Trade Liberalization and Productivity Growth. International Economic Review, v. 44, n. 4, p. 1383-1405, 2003.

Ferreira, P. C. Issler, J., Abreu S. P. Testing production functions used in empirical growth studies. Economics Letters 83 (1), 29-35, 2004.

Greene, W.H. Econometric Analysis; Prentice Hall, 5th edition (or higher), 2003.

Hausmann R., J. Hwang and D. Rodrik. 'What You Export Matters', Journal of Economic Growth, 12, 1-25, 2007.

Hausmann R. and D. Rodrik, 'Economic Development as Self Discovery', Journal of Development Economics, 72 (2), 603-633, 2003.

Hummels, D. and Klenow, P., 'The Variety and Quality of a Nation's Export', The American Economic Review, 95, 3, 704-723, 2005.

Jarreau, J.; Poncet, S. Export sophistication and economic performance: evidence from Chinese provinces. CEPII, 2009. (WorkingPaper, n. 34). 
Kume, Honório et al. O grau de sofisticação relativo das exportações brasileiras: 19962008. Texto para Discussão. Brasília, IPEA, 2012.

Lall S., J. Weiss and J. Zhang,. 'The "Sophistication" of exports: a new trade measure', World Development, 34 (2), 222-237, 2006.

Lucas, R. E. On the mechanic of economic development. Journal of Monetary Economics, v. 22, n. 1, p. 3-42, 1988.

Mankiw, N. G.; Romer, D.; Weil, D. N. A Contribution to the Empirics Economic Growth. Quarterly Journal of Economics, v. 107, p. 407-437, 1992.

Mehlum, H., K.Moene and R.Torvik, 'Institutions and The Resource Curse', The Economic Journal, 116, 1-20, 2006.

Nelson, R. R., Phelps, E. S. Investment in Humans, Technological Diffusion, and Economic Growth. American Economic Review, v. 56, n. 2, p. 69-82, 1966.

Rodrik D., 'What is so special about China's Exports?', China \& World Economy, 14 (5), 1-19, 2006.

Schott, P. 'The Relative Sophistication of Chinese Exports', Economic Policy, 1, 5-49, 2008.

Sterlacchini, A. 'R\&D, Higher Education and Regional Growth: Uneven Linkages Among European Regions', Research Policy, 37, 1096-1107, 2008.

Xuan et al, What Drives the Export Sophistication of Countries? Working Paper Series. University of Oxford. Nº33, 2007.

$\mathrm{Xu}, \mathrm{B}$; $\mathrm{Lu}, \mathrm{J}$. Foreign direct investment, processing trade, and the sophistication of China's exports. China Economic Review, n. 20, p. 425-439, 2010. 